\title{
How to prevent post-ESD esophageal stricture
}

\section{(2) $\circledast \Theta$}

\author{
Author \\ Jan Martinek \\ Institution \\ Department of Hepatogastroenterology, IKEM, Prague, \\ Czech Republic \\ Bibliography \\ DOI https://doi.org/10.1055/a-0889-8010 | \\ Endoscopy International Open 2019; 07: E771-E773 \\ (c) Georg Thieme Verlag KG Stuttgart · New York \\ eISSN 2196-9736
}

\author{
Corresponding author \\ Jan Martinek, Institut klinicke a experimentalni mediciny - \\ Hepatogastroenterology, Videnska 1958 Praha 14021, \\ Czech Republic \\ Fax: +00420261364016 \\ jan.martinek@volny.cz
}

In real life, progress often creates another problem. For example, antibiotics were a breakthrough as they allowed us to treat untreatable infections, but we have to cope with several problems associated with these drugs, such as pseudomembranous colitis or antibiotic resistance. Another example is airplanes, which are now more advanced and a lot more automatic, but the latest software may lead to pilot confusion and result in a disastrous accident.

Recent advances in therapeutic endoscopy are, in my view, one of the major changes in medicine - no doubt, the technology is epoch-making. This is especially true for patients with early digestive neoplasias. While 15 to 20 years ago, almost all of these patients were referred for surgery, today, a substantial proportion of them can be treated (and cured) endoscopically by using endoscopic resection, endoscopic submucosal dissection or endoscopic full-thickness resection techniques. As esophagectomy represents a major operation carrying a significant risk of mortality and morbidity, endoscopic treatment of early esophageal neoplasia represents a major benefit for these patients. The old paradigm has changed: patients with high-grade dysplasia or early esophageal cancer do not need to undergo esophagectomy as they can be treated by using mini-invasive endoscopy. Compared to surgery, endoscopic treatment carries a much lower risk of severe adverse events, and the mortality rate is near zero.

With endoscopic resection (ER) techniques experiencing unprecedented advance, it is possible to endoscopically remove very large superficial tumors. In the esophagus, tumors occupying the whole circumference are radically removable. However, there is a dark side of this progress. The major drawback of extensive resection of esophageal mucosa is stricture formation. If the extent of circumferential resection is more than $60 \%$ of esophageal circumference, risk of stricture rises to $70 \%$ to $80 \%$. In patients undergoing resection of the whole circumfer- ence, risk of stricture is almost $100 \%$, especially if the resection area comprises a long esophageal segment [1]. These strictures are often refractory, requiring several sessions of usually balloon dilatation, which increases risk of complications and decreases patient quality of live. Because of the high risk of strictures, a combination of ER (or endoscopic submucosal dissection) of a given lesion followed by ablation of Barrett's esophagus segment (usually radiofrequency ablation [RFA]) is currently considered the standard approach to patients with Barrett's esophagus-related neoplasia. However, providing that there is an effective and safe method for stricture prevention, radical en-bloc resection of the whole $B E$ segment could have an advantage compared with a currently ER/RFA recommended approach. For example, RFA treatment consists of several sessions, is expensive, and does not provide a specimen for histology.

At present, prevention of post-ESD esophageal stricture is an unresolved, or partially resolved, issue. Even though the majority of post-ER/ESD strictures are effectively treated with balloon or bougie dilatation, some may be quite resistant to therapy and dilatation is not free of severe complications. Thus, finding of an effective, simple and not expensive method for stricture prevention would be of significant benefit. Unfortunately, despite the multiple studies performed and approaches tested so far, an effective, safe, and simple method has not been found. On the other hand, some currently available preventive measures have proven at least partially effective and should be considered in patients with extensive ER/ESD to prevent stricture formation. Therefore, all patients undergoing extensive esophageal ESD should receive a prophylactic measure. The question remains, however, which method of stricture prevention is the most effective. Various methods of post-ER/ESD stricture prevention have been tested in experimental or human studies and can be categorized as: 
1. systemic administration of anti-inflammatory substances (usually steroids)

2. local injection (or lavage) of anti-inflammatory or antifibrogenic substances (most often steroids)

3. purely endoscopic methods (transitory stent placement, dilatation)

4. tissue engineering methods - cell-based therapies

5. various other approaches

Systemic steroids are relatively effective in preventing post-ESD stricture if the resection comprises less than $100 \%$ of the circumference (rate of strictures is around 20\%) and if a stricture develops, steroids make it less refractory; treatment requires less sessions of balloon dilatation [2,3]. Systemic steroids can be combined with either local steroids or with stenting, but there are no prospective studies assessing these approaches. In our center (IKEM, Prague), we usually administer high-dose steroids (starting at $150 \mathrm{mg}$ of prednisolone for 3 days, then $60-80 \mathrm{mg}$ for $31 / 2$ weeks, then $40 \mathrm{mg}$ for 2 weeks and then gradual tapering, with a total duration of steroid treatment of 8 weeks), usually without stent placement and the rate of strictures is around $20 \%$. The major disadvantage of systemic steroid is the risk of systemic adverse events.

Locally administered steroids represent a promising approach because they have no or minimal systemic effects. There are several studies examining their effect, including one small, randomized trial, usually with triamcinolone acetonide $(80-100 \mathrm{mg})$ [4-7]. The results are conflicting. Some studies report stricture rates as low as $10 \%$ (vs. $66 \%$ in controls), while other studies did not show a preventive effect [4, 7]. Importantly, intralesional steroid injection is not free of complication as one case of delayed esophageal perforation following injection of triamcinolone has been described [8]. However, a majority of studies administered triamcinolone once and the studies differ with regard to technique of injection as well as to dose of triamcinolone.

The current retrospective study [9] assessed the effectiveness and safety of two triamcinolone injections given 2 weeks apart. The authors (pioneers of this method of prevention) found that two triamcinolone injections significantly decreased the rate of strictures (45.7\% vs. $73.9 \%$ ) and the need for balloon dilatation in patients with sub-circumferential ESD, however, steroids did not significantly prevent a stricture in patients with circumferential ESD ( $80 \%$ vs. $100 \%, P=0.62)$, there was a trend for less balloon dilatations in the active group ( 7 $[0-11]$ vs. $13[6-19] P=0.96)$. The major strength of the study is the relatively high number of patients included and a clear analysis separating patients with circumferential vs. sub-circumferential ESD. The major limitations include a retrospective design with historical controls and no standardized follow-up protocol. It is not clear that all patients completed a follow-up, histology from the healed defects is missing, and details on medical and endoscopic treatments in patients undergoing balloon dilatation are not provided. Thus, this study cannot be considered as a key piece of evidence to recommend local injection (or injections) of triamcinolone as the preferred method of postESD stricture prevention. There is a need for a randomized study comparing different triamcinolone protocols including a new interesting approach without injection into the submucosa, but with an esophageal triamcinolone filling performed twice - just after ESD and 1 week later. A retrospective study showed very promising results [10]. In addition, trials comparing local steroid treatment with other methods (systemic steroids, stents, preventive dilatations, tissue engineering methods) or trials examining the effectiveness of treatment combinations (e.g. local steroids with stents) would be of interest. Importantly, studies should be carried out separately in patients with sub-circumferential ESD and in patients with circumferential ESD as sub-circumferential ESD does not represent such an enormous problem.

As mentioned above, there are more methods for stricture prevention but high-quality evidence for their effectiveness is not available. It concerns preventive balloon dilatation strategy (starting with dilatation prior to development of a stricture), esophageal stenting (fully covered metallic stents may be an option, and biodegradable stents seem inappropriate in this setting), tissue engineering methods - cell-based therapies (some effectiveness reported, but these methods are technically demanding and expensive). Several new approaches have been further tested, such as spraying of a hemostatic powder, mitomycin $\mathrm{C}, \mathrm{N}$-acetylcysteine, injection of botulinum toxin or small interfering RNA, use of stents covered with a special material (acellular biomatrix from pig's epidermis, stents covered with a human amniotic membrane), drug eluting stents (steroids), and transplantation of gastric mucosa to the site of after ESD. These methods are summarized in a review article [1] and may represent interesting approaches, however, more experimental and clinical studies (preferably of a better quality) are awaited. The main problem is that the number of patients undergoing extensive esophageal ESD is rather limited, therefore, it is not easy to set up a large study. Thus, multicenter (maybe international) collaboration should be considered.

\section{Acknowledgements}

Supported by a grant from IGA NV16-27653A (Czech Health Research Council).

Competing interests

None

References

[1] Martínek J, Juhas S, Dolezel R et al. Prevention of esophageal strictures after circumferential endoscopic submucosal dissection. Minerva Chir 2018; 73: 394-409

[2] Yamaguchi N, Isomoto H, Nakayama T et al. Usefulness of oral prednisolone in the treatment of esophageal stricture after endoscopic submucosal dissection for superficial esophageal squamous cell carcinoma. Gastrointest Endosc 2011; 73: 1115 - 1121

[3] Sato $\mathrm{H}$, Inoue $\mathrm{H}$, Kobayashi $\mathrm{Y}$ et al. Control of severe strictures after circumferential endoscopic submucosal dissection for esophageal 
carcinoma: oral steroid therapy with balloon dilation or balloon dilation alone. Gastrointest Endosc 2013; 78: 250 - 257

[4] Takahashi H, Arimura Y, Okahara S et al. A randomized controlled trial of endoscopic steroid injection for prophylaxis of esophageal stenoses after extensive endoscopic submucosal dissection. BMC Gastroenterol 2015; 15: 1

[5] Hashimoto S, Kobayashi M, Takeuchi M et al. The efficacy of endoscopic triamcino- lone injection for the prevention of esophageal stricture after endoscopic submucosal dissection. Gastrointest Endosc 2011; 74: 1389-1393

[6] Hanaoka N, Ishihara R, Takeuchi Y et al. Intralesional steroid injection to prevent stricture after endoscopic submucosal dissection for esophageal cancer: a controlled prospective study. Endoscopy 2012; 44: 1007 - 1011
[7] Hanaoka N, Ishihara R, Uedo N et al. Refractory strictures despite steroid injection after esophageal endoscopic resection. Endosc Int Open 2016; 4: E354-E359

[8] Yamashina T, Uedo N, Fujii M et al. Delayed perforation after intralesional triamcinolone injection for esophageal stricture following endoscopic submucosal dissection. Endoscopy 2013; 45: E92

[9] Hashimoto S, Mizuno K, Takahashi K et al. Evaluating the effect of injecting triamcinolone acetonide in two sessions for preventing esophageal stricture after endoscopic submucosal dissection. Endosc Int Open 2019; 07: E764-E770

[10] Ishimura N, Oshima N, Mishiro T et al. Esophageal triamcinolone acetonide-filling method: a novel procedure to prevent stenosis after extensive esophageal endoscopic submucosal dissection. Shibagaki K, Gastrointest Endosc 2018; 87: 380 - 389 